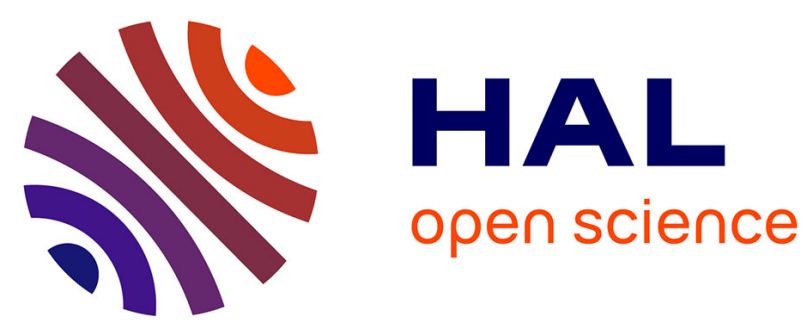

\title{
How to Catch a Smurf? - Ageing and Beyond...In vivo Assessment of Intestinal Permeability in Multiple Model Organisms
}

Raquel Martins, Andrew John Mccracken, Mirre Simons, Catarina Henriques, Michael Rera

\section{To cite this version:}

Raquel Martins, Andrew John Mccracken, Mirre Simons, Catarina Henriques, Michael Rera. How to Catch a Smurf? - Ageing and Beyond...In vivo Assessment of Intestinal Permeability in Multiple Model Organisms. Bio-protocol , 2018, 8 (3), 10.21769/BioProtoc.2722 . hal-02347578

\section{HAL Id: hal-02347578 \\ https://hal.science/hal-02347578}

Submitted on 16 Nov 2021

HAL is a multi-disciplinary open access archive for the deposit and dissemination of scientific research documents, whether they are published or not. The documents may come from teaching and research institutions in France or abroad, or from public or private research centers.
L'archive ouverte pluridisciplinaire HAL, est destinée au dépôt et à la diffusion de documents scientifiques de niveau recherche, publiés ou non, émanant des établissements d'enseignement et de recherche français ou étrangers, des laboratoires publics ou privés. 


\title{
How to Catch a Smurf? - Ageing and Beyond...
} In vivo Assessment of Intestinal Permeability in Multiple Model Organisms Raquel R. Martins ${ }^{1,2}$, Andrew W. McCracken 2, 3 , Mirre J. P. Simons ${ }^{2,3}$, Catarina M. Henriques ${ }^{1,2}$ and Michael Rera ${ }^{4, *}$

\footnotetext{
${ }^{1}$ Department of Oncology and Metabolism, Medical School, University of Sheffield, Sheffield, UK; ${ }^{2}$ Bateson Centre, University of Sheffield, Sheffield, UK; ${ }^{3}$ Department of Animal and Plant Sciences, University of Sheffield, Sheffield, UK; ${ }^{4}$ Unité de Biologie Fonctionnelle et Adaptative (BFA) UMR8251 CNRS - Université Diderot, Sorbonne Paris Cité, Paris, France

*For correspondence: michael.rera@univ-paris-diderot.fr
}

\begin{abstract}
[Abstract] The Smurf Assay (SA) was initially developed in the model organism Drosophila melanogaster where a dramatic increase of intestinal permeability has been shown to occur during aging (Rera et al., 2011). We have since validated the protocol in multiple other model organisms (Dambroise et al., 2016) and have utilized the assay to further our understanding of aging (Tricoire and Rera, 2015; Rera et al., 2018). The SA has now also been used by other labs to assess intestinal barrier permeability (Clark et al., 2015; Katzenberger et al., 2015; Barekat et al., 2016; Chakrabarti et al., 2016; Gelino et al., 2016). The SA in itself is simple; however, numerous small details can have a considerable impact on its experimental validity and subsequent interpretation. Here, we provide a detailed update on the SA technique and explain how to catch a Smurf while avoiding the most common experimental fallacies.
\end{abstract}

Keywords: Smurf Assay, Digestive tract permeability, Blue dye \#1, Ageing

[Background] The Smurf Assay (SA) is based on the Drosophila feeding assay described in (Wong et al., 2009). The assay assesses food intake by the co-ingestion of a blue dye, which is not absorbed by the digestive tract, thereby allowing direct quantification. For the SA it is essential that this specific blue dye does not pass an intact intestinal barrier since its readout is a whole body coloration (here blue). This property allows direct in vivo assessment of gut permeability, which has been shown to increase with age (Rera et al., 2011 and 2012).

As recently discussed in Rera et al. (2018), the Smurf Assay is not only a simple way to assess intestinal permeability in vivo, but also an elegant way to assess the physiological age of individuals in a broad range of organisms. As such, it allows novel approaches to study the various events occurring in aging individuals (Tricoire and Rera, 2015; Rera et al., 2018).

In recent years, we have received numerous comments and questions about the initial protocol, leading us to develop the present extended protocol.

Specifics of the dye used: The dye typically used is FD\&C blue dye \#1, but we have also validated the use of red \#40 and fluorescein (Rera et al., 2011 and 2012). We adapted the use of the very same blue \#1 to zebrafish (Dambroise et al., 2016) and killifish (Rera et al., 2018) but found it is easier to use fluorescein with nematodes although the same blue \#1 can also be used as demonstrated in (Gelino et 
al., 2016). The dye is non-toxic and does not decrease the lifespan of individuals when exposed during their whole life (Figure 1A). Moreover, no reduction in longevity is detected even when the gut becomes permeable and the dye diffuses into the body, contrary to what was recently suggested in Clark et al. (2015). We confirmed this by placing newly identified Smurfs on normal non-dyed media, and this did not lead to a longer lifespan (Figure 1B).
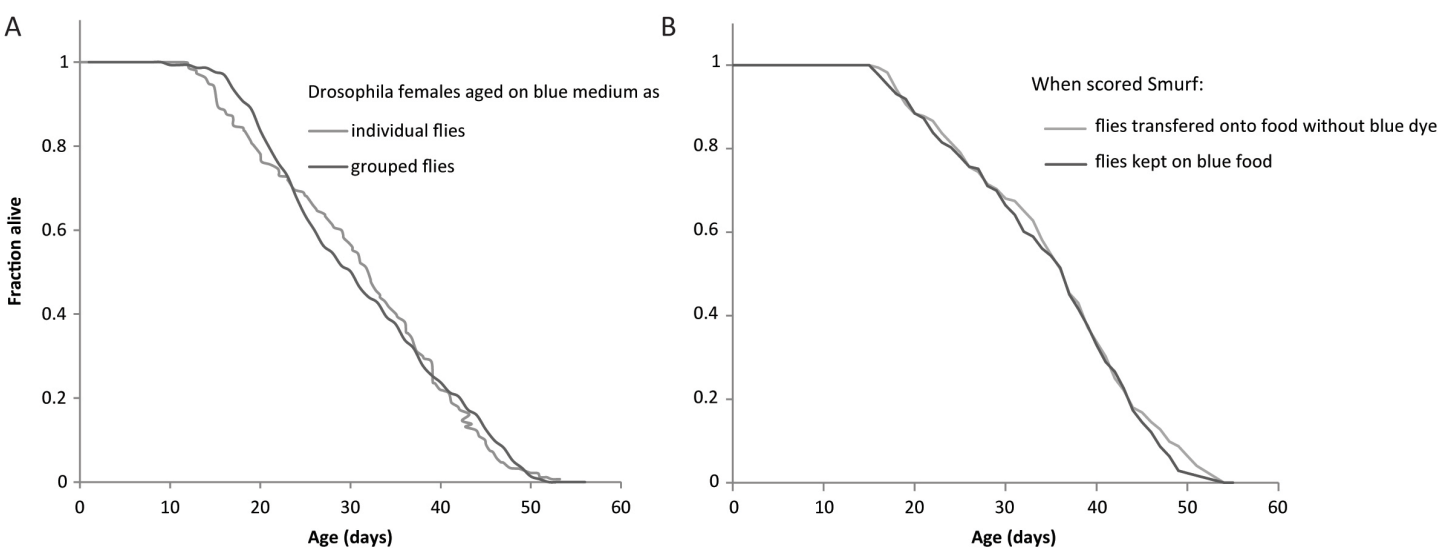

Figure 1. The blue dye \#1 is not toxic neither for non-Smurfs or Smurfs. A. The longevity curve of 1,146 individual female flies maintained on blue medium overlaps the longevity curves of 295 female flies maintained on standard medium by groups of 28-32 individuals (longevity data from Tricoire and Rera, 2015). B. The longevity curve of 173 individual female flies maintained on blue dye for their whole lives overlaps the longevity curve of 172 individual female flies transferred back to standard medium when they became Smurf. This confirms that Smurfs do not die prematurely because the dye gains toxic properties when it diffuses through the gut.

\section{Catching Smurfs}

Although we initially described Smurfness as a well-marked, almost binary phenotype (Rera et al., 2011 and 2012; Tricoire and Rera, 2015), Smurfness is, as most phenotypes are, continuous (Figures 2A-2D, Clark et al., 2015). Thus, it is important to understand that the lighter the Smurf is, the greater the chance of misidentifying Smurf individuals. Indeed, the major part of uncertainly identified Smurfs appears in the few days preceding clear mortality acceleration in the population (Figure 3). The continuous nature of the Smurf phenotype can have two main causes. First, the dye might take some time to diffuse through limited gut permeability, thus generating a determined relationship between Smurfness and the level of gut permeability. Second, there can be biological (environmental and/or genetic factors) that can cause variation in the phenotype observed.

Moreover, there can be observer bias, attributable to the experimenter who is sorting and classifying individuals. We noticed that the earlier in the lifespan, and the fewer Smurfs are present in the group, the more likely an observer is to classify individuals as Smurfs, despite subsequently being scored as non-Smurf. The latter is probably inherent in the way we distinguish individuals based on their surrounding individuals, and hence the more Smurf individuals are present, the more stringent we are 
on their identification. To circumvent this, single individuals could be photographed for independent verification. In practice, however, it is difficult to both sort large numbers of flies and photograph every individual for subsequent blue hue quantification. This problem is less relevant to larger organisms.
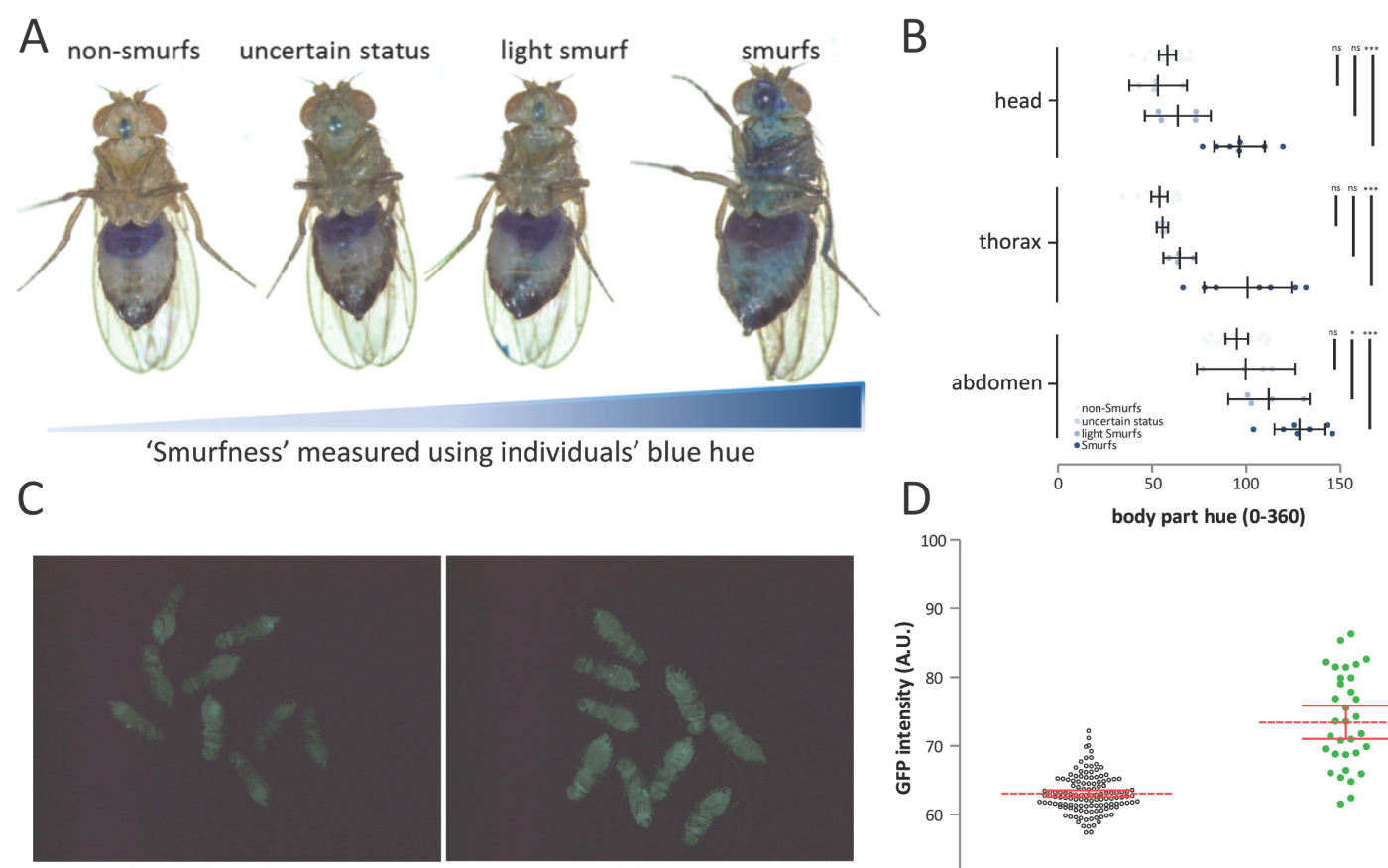

'Smurfness' measured using individuals' fluorescence

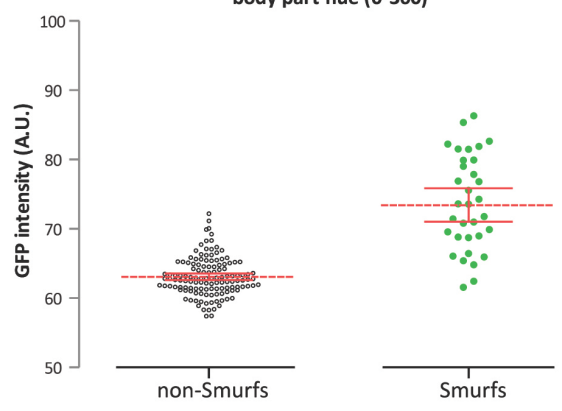

Figure 2. The Smurf phenotype is not binary but rather continuous. A. The continuous aspect of the Smurf phenotype was previously described in Clark et al. (2015), but we noticed much more subtle shades of blue in our experimental conditions. B. Nevertheless, only the two categories of Smurfs and non-Smurfs showed significant blue hue difference on all body parts ( $n=31$ female from the drsGFP genotype, $n_{n s}=16, n_{?}=4, n_{1 s}=4$ and $n_{s}=7$ )-a subsequent experiment with larger $\mathrm{n}$ was conducted and showed significant differences only between Smurfs and non-Smurfs (not shown). C. The continuous Smurfness distribution is not just due to the Smurf (blue dye based) assay but is also observable in the drsGFP individuals by D. measuring GFP intensity in Smurfs ( $\mathrm{n}$ $=33$ ) and non-Smurfs $(n=130)$. The drosomycin promoter-driven GFP expression has been shown to be a surrogate of Smurfness in Rera et al. (2012). Mated 35-40 days old female Drosophila. 


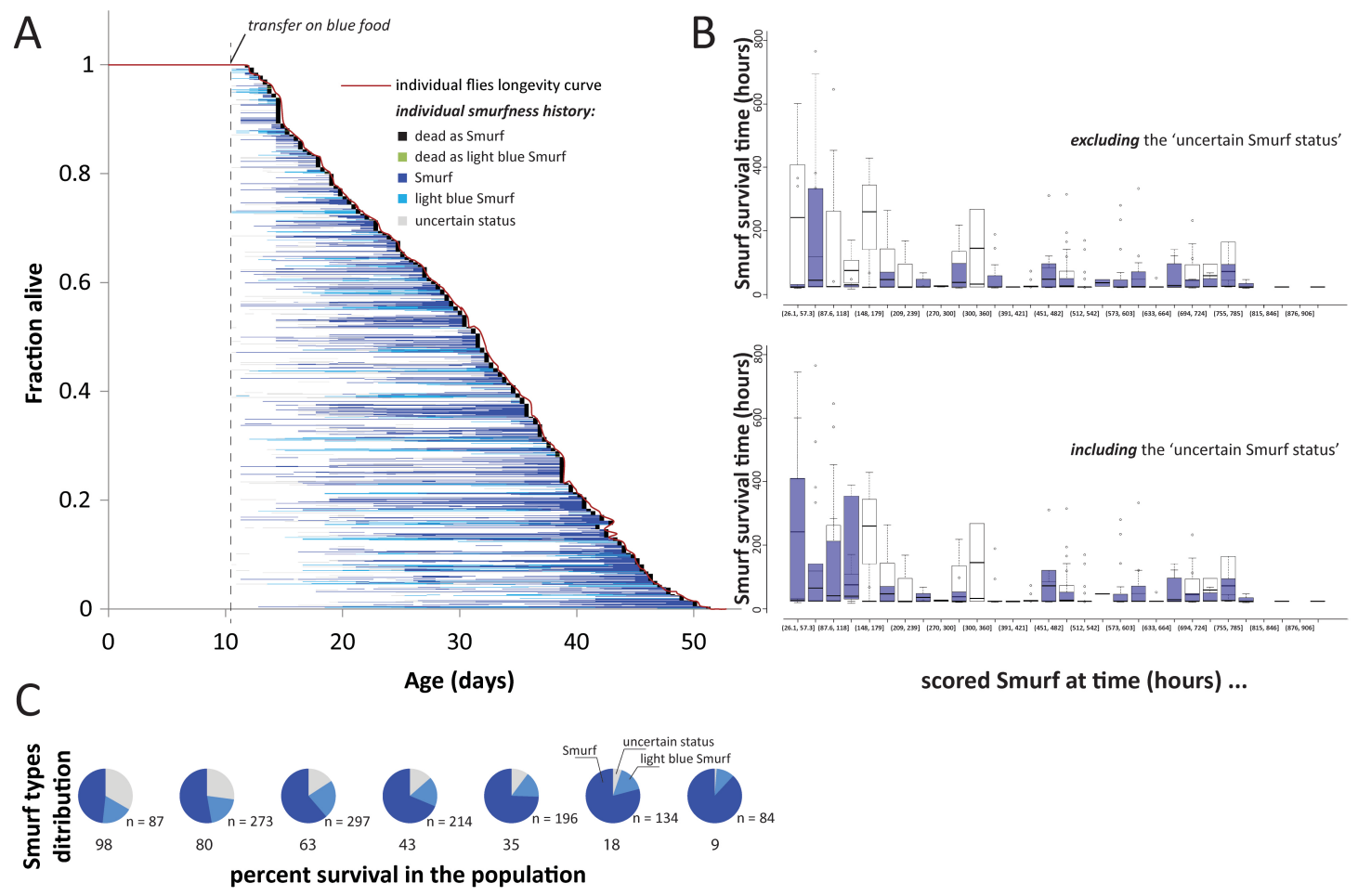

Figure 3. All individuals eventually become Smurf prior to death. Estimating Smurf survival time requires taking into account individuals from different moments of the survival experiment to prevent misestimation. A. All 1,146 individual female flies became Smurf prior to death and survived for various duration in that state. B. The uncertainty on the Smurf status has the strongest effect on the youngest identified Smurfs. Restricting Smurf studies to that period is thus at high risk of misestimating their remaining lifespan. We recommend to study them close to the $T_{50}$ of the population. Original data from (Tricoire and Rera, 2015). C. Proportion of the three different types of living Smurfs at various percent survival in the population. The largest population of uncertain Smurf individuals is restricted to the first few days of Smurf apparition in the population.

\section{Other experimental considerations}

The duration of exposure to the dye does not affect survival nor the Smurf Increase Rate (SIR). For ease we now use overnight feeding on the blue dye. The fly population density is of critical importance: we observed that at a too high density, individuals tend to get covered with blue faeces. Although easy to rinse with water (the addition of some ethanol can help immersion) to discern 'false' Smurfs from legitimate ones, we do not recommend more than 30 individuals per vial for overnight exposure. For continued exposure to the dye, lower numbers should be considered. Moreover, when learning how to distinguish smurfs we recommend washing flies to confirm the phenotype. These considerations could be especially important as behaviour and the quantity of feaces produced can differ between genetic backgrounds and experimental conditions.

We received a significant number of questions regarding 'the number of Smurfs with time'. As previously stated in (Rera et al., 2011 and 2012; Tricoire and Rera, 2015; Dambroise et al., 2016), it is 
the Smurf proportion calculated as $\frac{n_{\text {Smurfs }}}{n_{\text {Smurfs }}+n_{\text {non-Smurfs }}}$ at a specific age that increases as a function of age, rather than the absolute number of Smurf individuals. The interpretation of this number is similar to that of mortality risk, as it is related to the age-specific risk of an individual in the population to become a Smurf. Note that Smurfs remain in the population for a short time until they die and thus remain in the numerator of the formula above. The Smurf proportion is thus not equal to the risk of becoming a Smurf, but could be calculated as such (Promislow et al., 1999).

Most of the Smurf-related studies we conduct are based on female flies because, as we described in (Rera et al., 2012), they are easier for Smurf identification, principally since their abdomen is larger. In addition, the age-dependent SIR is weaker in males (see Figure S1A in Rera et al., 2012). This might be due to a much shorter remaining lifespan of males when they are in the Smurf state, as we recently observed (unpublished) and/or their smaller body. It is interesting to notice that in zebrafish the sexspecific SIR intensity was inverted (see Figure S1B in Dambroise et al., 2016). Regardless, male Drosophila do undergo the Smurf transition prior to death (Figure 4), contrary to what was recently suggested in Regan et al., 2016.
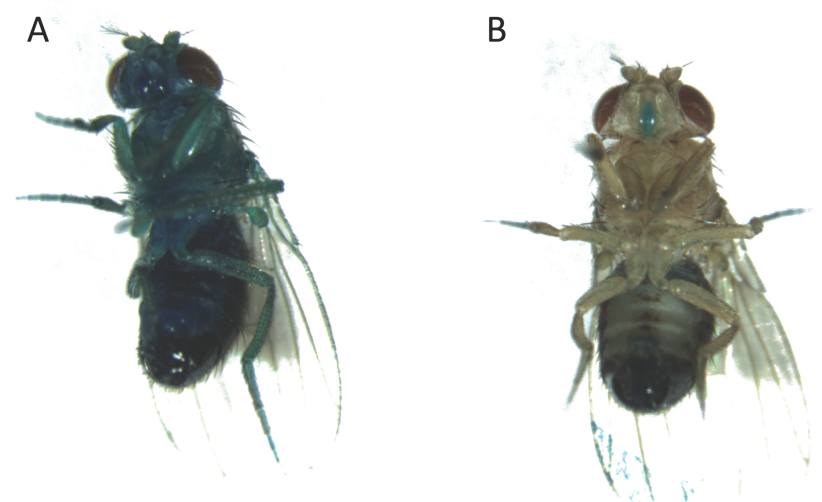

Figure 4. The Smurf phenotype occurs in male Drosophila melanogaster. Two examples are pictured: A. A smurf male; B. A non-smurf male. 35 days old males.

\section{Materials and Reagents}

1. Parafilm

2. $0.22 \mu \mathrm{m}$ sterile vacuum filter (Corning ${ }^{\circledR}$ bottle-top vacuum filter, Corning, catalog number: 430015)

3. $0.45 \mu \mathrm{m}$ filter (VWR, catalog number: 514-4127)

Note: The $0.45 \mu \mathrm{m}$ filter is for fish.

4. $30 \mathrm{G}$ syringe (BD, catalog number: 324826)

5. Narrow plastic vials

6. 22-G Micro-Renathane Implantation tubing (Braintree Scientific, catalog number: MRE025)

7. Disposable syringe $0.3 \mathrm{ml} \mathrm{BD}$ needle $30 \mathrm{G}$ (Insuline) (BD, catalog numbers: 324826,320837 ) 
8. Soft sponge of approximately $20 \mathrm{~mm}$ in height (such as Jaece Industries, catalog number: L800D)

9. Drosophila and fish (any source)

10. FD\&C blue dye \#1 (Sigma-Aldrich, catalog number: 861146, SPS Alfachem Ref: 101-2912 and A \& Z Food Additives Brilliant Blue FCF(CAS No. 3844-45-9), FD\&C Blue 1, E133)

11. Fluorescein sodium salt (Sigma-Aldrich, catalog number: F6377-500g)

12. FD\&C Red \#40, Allura Red (SPS Alfachem)

13. Bi-distilled deionized water $\left(\mathrm{ddH}_{2} \mathrm{O}\right)$

14. Hanks' balanced salt solution (HBSS, Thermo Fisher Scientific, Gibco ${ }^{\mathrm{TM}}$, catalog number: 14175053)

15. Buffered tricaine methanesulfonate (Sigma-Aldrich, catalog number: A5040) $164 \mathrm{mg} / \mathrm{L}$ in fish tank water

16. Moldex (VWR, catalog number: 25605.293)

17. Blue \#1 stock solution (12.8x) (see Recipes)

18. Dyed media (see Recipes)

\section{Equipment}

1. Magnetic stirrer

2. $5 \mathrm{~L}$ glass beaker

3. $2 \mathrm{~L}$ glass bottle

4. LED cool white lighting

5. White background

6. Epi-fluorescence microscope Nikon Eclipse 80i for nematode Smurfs (Nikon, model: Eclipse 80i)

\section{Software}

1. ImageJ 1.46j and above

2. GraphPad Prism 6.01 


\section{Procedure}

A. Prepare solutions

See 'Recipes' section.

B. Smurf assay

Notes:

a. All fish work was performed following Animals (Scientific Procedures) Act 1986 and UK Home Office approval (PPL 70/8681).

$b$. If the blue dye is used at the correct concentration, well dissolved and filtered, no adverse effects are expected from the 30 min incubation period, except some temporary stress and discomfort. After blue-dye incubation, fish should be kept in separate tanks for at least $4 \mathrm{~h}$ before joining the main aquarium tanks where water is shared, to ensure all dye is excreted.

c. Any signs of potential adverse effects such as impaired prolonged swimming and feeding should be brought to the attention of the NACWO and/or NVS and any definite or probably adverse effects should lead culling of the animal using an approved procedure performed by a person registered as competent by the certificate/licensed holder (10.1089/zeb.2016.1248).

d. Addendum by Jennifer C. Regan and Michael Rera

One question that we have not fully highlighted in the present protocol is how much the environmental conditions can affect the Smurf phenotype. As the association between Smurfness and death is strong, any condition able to affect lifespan-genotype, food recipe, local gut microbiota, Wolbachia infection status, etc.-is able to affect the proportion of Smurfs at a given time. This is why we tried to emphasize the need for considering the percent survival of population rather than absolute chronological time for the comparison of Smurf proportion in different labs.

One example of this variability is the low proportion of Smurfs obtained in an outbred line, wDahomey (Regan et al., 2016), in which the barrier phenotype was suppressed such that a low proportion of females, and very few Smurf males (if any) were recovered in the experimental setup. As an example of the combined effect of genotype and environmental conditions, inbred $\left(w^{1118}\right)$ flies reared in the same lab produced a higher proportion of Smurfs, including a significant proportion of males (Supp. data of Regan et al. (2016)).

N.B. This has been presented in a way that is unfortunately easy to misinterpret as Smurfs not appearing at all in males (Regan et al., 2016). However, this was not the case and indeed, the observed dimorphism appears to be consistent between labs (Rera et al., 2012; Dambroise et al., 2016; Regan et al., 2016).

It is important to standardize the conditions under which Smurfs are examined.

1. Transfer individuals onto SA medium

a. Drosophila and nematodes: overnight.

b. Fish: 30 min. 
2. Transfer individuals onto fresh normal medium for immediate scoring

a. Drosophila: if you have to process large numbers of vials, you might want to transfer vials one at a time to prevent individuals from clearing the blue dye before scoring.

b. Nematodes can be examined directly on the fluorescein medium using an epi-fluorescence microscope.

c. Fish: rinse them until no blue comes out of the individuals in successive and independent fish tanks (Figure 5).
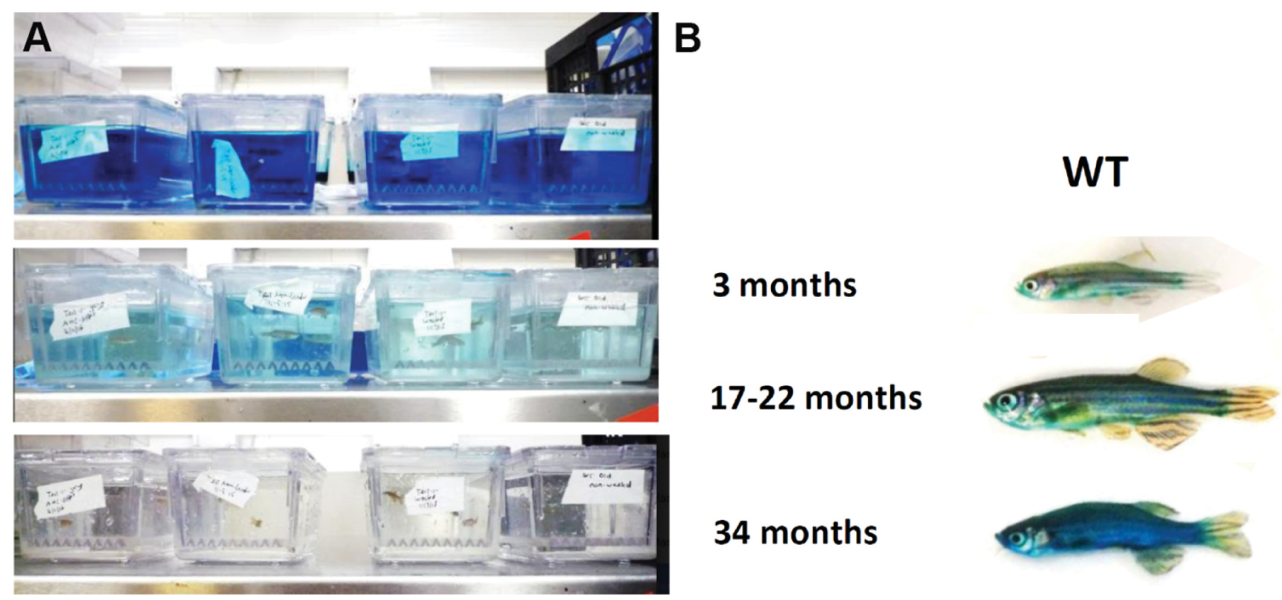

Figure 5. Identifying Smurfs in zebrafish using the 'bathing protocol'. A. Successive fish tanks are used to rinse individuals until no further dye is emitted. B. Representative images of Smurf and non-Smurf individuals in WT AB zebrafish.

3. Count individuals that are positive for Smurfness (SA+) as well as those that are negative (SA-). The proportion of Smurfs in the population is then $p=\frac{S A+}{S A++S A-}$

4. Smurf and non-Smurf individuals can then be monitored separately for further analysis.

An alternative protocol for fish (Raquel Martins and Catarina Henriques): Since penetration of the blue dye through the skin of fish will always be a confounding factor with a dipping protocol, we tested oral administration of the blue dye by following the adult zebrafish gavage protocol published in Collymore et al. (2013). $5 \mu \mathrm{l}$ of a $5 \%(\mathrm{~m} / \mathrm{v})$ filtered blue dye dissolved in Hanks' balanced salt solution (HBSS) was administered through oral gavage. Older individuals show the Smurf phenotype whilst we did not observe this in younger individuals (Figure $6 \mathrm{C}$ ). This method requires training to prevent the experimenter from injuring the gut. It is nevertheless non-lethal, thus allowing further monitoring of individuals post assay. 


\section{A Oral gavage}

Blue dye in HBSS

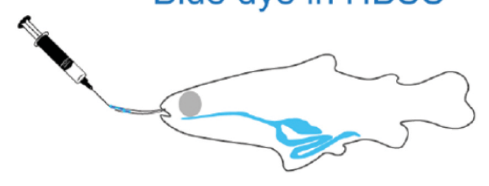

B

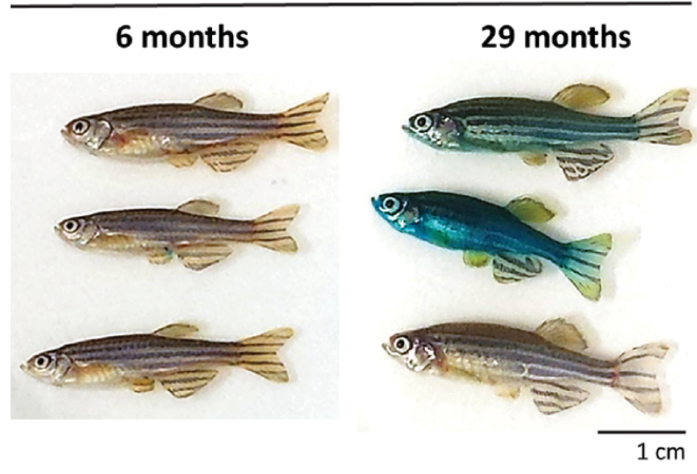

C Blue hue

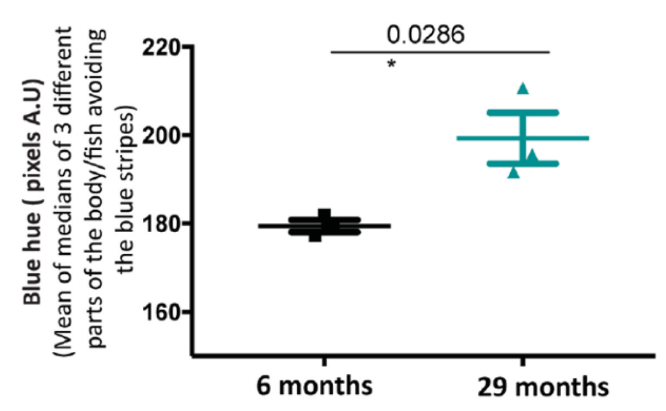

Figure 6. Gavage allows to identify zebrafish Smurfs. Smurf zebrafish obtained using the 'gavage protocol' (A). All six Zebrafish WT AB individuals of 6 and 29 months of age used (B) and respective quantification of the blue hue that leaked through the gut and is now visible on the skin. (C) Quantifications were performed from digital pictures in ImageJ selecting three different parts of the body/fish, avoiding the blue stripes and quantifying the median of pixels per area. All 3 older fish turned bluer than the younger fish ( $t$-test assuming normality and equal variances: $P<0.05$ or more conservative: unequal variance $t$-test or non-parametric Wilcoxon rank test $P \leq 0.10)$. See Video 1 .

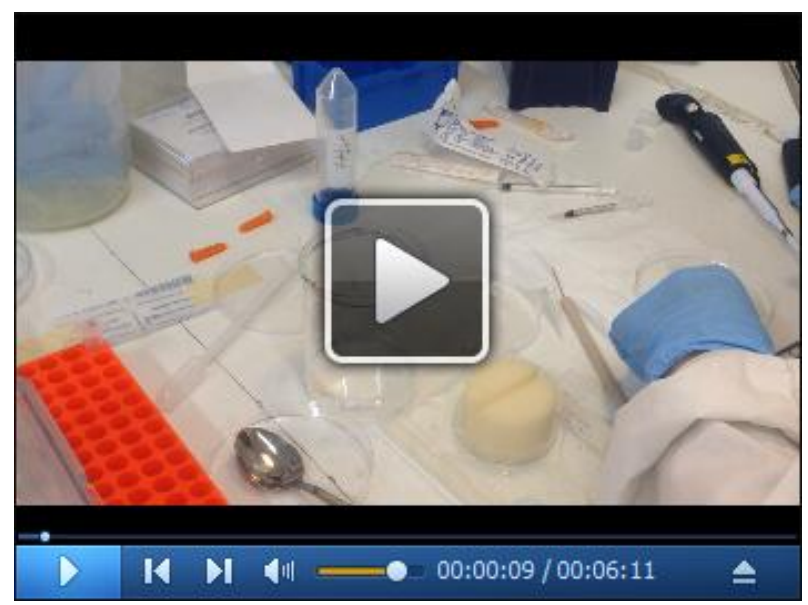

Video 1. Detailed zebrafish Smurf gavage protocol 


\section{Pre-injection preparation:}

a. Before the gavage procedure, the fish must be fasted for $24 \mathrm{~h}$ or at least $4 \mathrm{~h}$ prior to gavage, which will empty the intestinal bulb (stomach) contents.

b. On the flat face, make a cut (10-15 mm deep) in the soft sponge, which will hold the fish for injection.

c. Then, set the sponge into a $60 \mathrm{~mm}$ Petri dish. This will hold water to help maintain sponge moist.

\section{Anesthesia, Injection and Recovery:}

a. Anaesthetize fish with diluted Tricaine and monitor fish behavior. When fish is quiet, transfer it to the wet sponge (soaked in Tricaine) and move the sponge into a vertical position, as illustrated in the video.

b. Then, open the zebrafish mouth using the 22-G catheter tubing adjusted to the size of the fish (approximately $2.5 \mathrm{~cm}$ ), and gently insert the tubing until the tip is past the gills (approximately $1 \mathrm{~cm}$ or the length of the tubing). The implantation tubing should not need to be forced. Resistance suggests the tube may be hitting the gill arch or heart. Thus, if there is resistance, gently withdraw, reposition and try again.

c. When the tube is completely inserted (reaching the middle intestine), inject the material slowly ( $5 \mu$ of filtered $(0.45 \mu \mathrm{m}$ syringe filter) $5 \%(\mathrm{w} / \mathrm{v})$ blue \#1 diluted in HBSS). To accurately measure $5 \mu \mathrm{l}$, pipette $5 \mu \mathrm{l}$ of the solution onto Parafilm and aspirate with a syringe, avoiding air bubbles. While injecting, make sure that the solution does not exit via the gills or the mouth.

d. After injecting all the content, remove the fish from the sponge and place into the recovery tank. Fish should be monitored for regurgitation as shown by visualizing the fish actively expelling material from its mouth, or no opercular movement. Fish can be returned to their regular tank, or kept separately in a mating box, once they have recovered.

C. Blue hue measurements

Using ImageJ freehand selection tool, ROIs are selected on a picture converted to HSB space (command Image $\rightarrow$ Type $\rightarrow$ HSB Stack). Then, the hue is measured by the mean gray value (Analyze $\rightarrow$ Set measurements... $\rightarrow$ Mean gray value) in the Hue dimension of the picture.

\section{Data analysis}

It is possible to consider two distinct approaches for comparing the Smurf proportion in condition A versus condition $B$.

1. At one given time-point, comparing the proportion of Smurfs between conditions $A$ and $B$ : 
a. Use a two-sided binomial test to estimate the statistical significance of the difference between distribution of $\mathrm{SA}+$ individuals amongst the total population, in condition $\mathrm{B}$ compared to the distribution in control condition A. We used this approach in (Rera et al., 2011). Since GraphPad Prism Chi-square is just an approximation, one can use the exact test implemented in Excel or $\mathrm{R}$.

b. Use a Mann-Whitney test on the average proportion of SA+ individuals per vial in condition A and B.

2. Across multiple time-points, comparing the slope and y-intercept of age-dependent proportion of Smurfs between conditions A and B. We initially described this approach in (Rera et al., 2012) using GraphPad Prism. The calculations follow a method spelled in Chapter 18 of $\mathrm{J}$ Zar, Biostatistical Analysis, 2nd edition, Prentice-Hall, 1984. It is equivalent to analysis of covariance (ANCOVA). Similar approaches are available in mixed models, in a binomial model context and should be considered if one wants to correct for experimental confounds, such as batch or other random terms (Gelman et al., 2004).

We recommend the use of method 2 since the time trend ensures a smaller risk of false positives.

\section{Notes}

1. It is crucial to ensure that the bathing solution for fish does not contain clumps of the blue dye powder as they tend to stick to scales, making it harder to distinguish Smurf individuals.

2. Standardize your Smurf scoring conditions.

3. Train multiple people to recognize Smurf individuals and cross-check your own scoring with them.

4. Keep in mind that the genetic background of the individuals you assess for Smurfness can influence its result, so adapt your scoring to it.

\section{$\underline{\text { Recipes }}$}

The FD\&C blue dye \#1 comes as an extremely volatile dark purple powder. The first step is to put it in an aqueous solution. The maximum concentration we could reach at room temperature is $320 \mathrm{~g}$ $\mathrm{L}^{-1}$. This corresponds to a $12.8 \mathrm{x}$ stock solution as the final concentration in the medium is $2.5 \mathrm{~g}$ per $100 \mathrm{ml}(2.5 \% \mathrm{w} / \mathrm{v})$.

1. Blue \#1 stock solution (12.8x)
a. Pour $700 \mathrm{ml} \mathrm{dH_{2 }} \mathrm{O}$ into a $5 \mathrm{~L}$ glass beaker
b. Weigh $400 \mathrm{~g}$ of the blue \#1 powder
c. Gently pour the powder into the water containing beaker
d. Add the magnetic stirring bar
e. Cover the beaker with Parafilm
f. Stir until the powder is totally dissolved (typically a couple of hours) 
g. Slowly rinse the walls of the beaker with the remaining $300 \mathrm{ml} \mathrm{of} \mathrm{dH}_{2} \mathrm{O}$

h. Stir for $30 \mathrm{~min}$

i. Vacuum filter the blue solution into a sterile bottle

Notes:

i. For fish: prepare the $1 x$ solution directly in water extracted from the fish tank and filter it.

ii. For fluorescein: use the same procedure as for the blue \#1.

iii. The solution is preferentially used immediately or frozen and not stored at room temperature since its dark color does not allow the observation of any potential contamination.

2. Dyed medium

a. Prepare your standard Drosophila food recipe (Rera et al., 2011; Katzenberger et al., 2015; Tricoire and Rera, 2015; Barekat et al., 2016; Regan et al., 2016) with 7.8\% less water

b. After boiling the medium, add the Moldex (VWR)

c. Add $7.8 \mathrm{ml}$ of the Blue \#1 stock solution per $100 \mathrm{ml}$ (final) of food

d. Stir thoroughly until the coloration is homogenous

e. Dispense in the narrow vials (minimum $1.25 \mathrm{ml}$ for overnight SA) Notes:

i. For nematodes: use the same procedure as for the blue \#1. Although the molar mass of fluorescein is 2.9 times less than that of blue \#1 at that concentration the fluorescein does not fluoresce. This allows an easy identification of nematode fluorescent Smurfs directly on the medium.

ii. The fluorescein solution is added directly to the agar plate or mixed with the bacteria.

\section{Acknowledgments}

This work was supported by the CNRS to M.R. This protocol is adapted from (Rera et al., 2012; Dambroise et al., 2016). M.J.P.S. is supported by a Sir Henry Wellcome and a Sheffield University Vice Chancellor's Fellowship and the Natural Environment Research Council (N013832). A.W.McC. is supported by the NERC ACCE Doctoral training program. C.M.H is supported by a Sir. Henry Dale Fellowship by the Wellcome Trust \& Royal Society and a Sheffield University Vice Chancellor's Fellowship. R.R.M is supported by a Sheffield University Doctoral Academy PhD Fellowship. The authors declare no conflict of interest nor competing interests.

We want to thank Dr. Jennifer C. Regan for contacting us to express her concern regarding a comment made in our original manuscript. It helped starting a constructive discussion that led to the addition of Note d in Procedure B. 


\section{$\underline{\text { References }}$}

1. Barekat, A, Gonzalez, A., Mauntz, R. E., Kotzebue, R. W., Molina, B., El-Mecharrafie, N., Conner, C. J., Garza, S., Melkani, G. C., Joiner, W. J., Lipinski, M. M., Finley, K. D. and Ratliff, E. P. (2016). Using Drosophila as an integrated model to study mild repetitive traumatic brain injury. Sci Rep 6: srep25252.

2. Chakrabarti, S., Dudzic, J. P., Li, X., Collas, E. J., Boquete, J. P. and Lemaitre, B. (2016). Remote control of intestinal stem cell activity by haemocytes in Drosophila. PLoS Genet 12(5): e1006089.

3. Clark, R. I., Salazar, A., Yamada, R., Fitz-Gibbon, S., Morselli, M., Alcaraz, J., Rana, A., Rera, M., Pellegrini, M., Ja, W. W. and Walker, D. W. (2015). Distinct shifts in microbiota composition during Drosophila aging impair intestinal function and drive mortality. Cell Rep 12(10): 16561667.

4. Collymore, C., Rasmussen, S. and Tolwani, R. J. (2013). Gavaging adult zebrafish. J Vis Exp (78).

5. Dambroise, E., Monnier, L., Ruisheng, L., Aguilaniu, H., Joly, J. S., Tricoire, H. and Rera, M. (2016). Two phases of aging separated by the Smurf transition as a public path to death. Sci Rep 6: 23523.

6. Gelino, S., Chang, J. T., Kumsta, C., She, X., Davis, A., Nguyen, C., Panowski, S. and Hansen, M. (2016). Intestinal autophagy improves healthspan and longevity in C. elegans during dietary restriction. PLoS Genet 12(7): e1006135.

7. Gelman, A., Trevisani, M., Lu, H. and van Geen, A. (2004). Direct data manipulation for local decision analysis as applied to the problem of arsenic in drinking water from tube wells in Bangladesh. Risk Anal 24(6): 1597-1612.

8. Katzenberger, R. J., Chtarbanova, S., Rimkus, S. A., Fischer, J. A., Kaur, G., Seppala, J. M., Swanson, L. C., Zajac, J. E., Ganetzky, B. and Wassarman, D. A. (2015). Death following traumatic brain injury in Drosophila is associated with intestinal barrier dysfunction. Elife 4.

9. Promislow, Tatar, Pletcher and Carey (1999). Below-threshold mortality: implications for studies in evolution, ecology and demography. $J$ Evol Biol 12: 314-328.

10. Regan, J. C., Khericha, M., Dobson, A. J., Bolukbasi, E., Rattanavirotkul, N. and Partridge, L. (2016). Sex difference in pathology of the ageing gut mediates the greater response of female lifespan to dietary restriction. Elife 5: e10956.

11. Rera, M., Bahadorani, S., Cho, J., Koehler, C. L., Ulgherait, M., Hur, J. H., Ansari, W. S., Lo, T., Jr., Jones, D. L. and Walker, D. W. (2011). Modulation of longevity and tissue homeostasis by the Drosophila PGC-1 homolog. Cell Metab 14(5): 623-634.

12. Rera, M., Clark, R. I. and Walker, D. W. (2012). Intestinal barrier dysfunction links metabolic and inflammatory markers of aging to death in Drosophila. Proc Natl Acad Sci U S A 109(52): 21528-21533. 
13. Rera, M., Vallot, C. and Lefrancois, C. (2018). The Smurf transition: new insights on ageing from end-of-life studies in animal models. Curr Opin Oncol 30(1): 38-44.

14. Tricoire, H. and Rera, M. (2015). A new, discontinuous 2 phases of aging model: Lessons from Drosophila melanogaster. PLoS One 10(11): e0141920.

15. Wong, R., Piper, M. D., Wertheim, B. and Partridge, L. (2009). Quantification of food intake in Drosophila. PLoS One 4(6): e6063. 